\title{
ICD-11 Prolonged Grief Disorder Criteria: Turning Challenges Into Opportunities With Multiverse Analyses
}

\author{
Maarten C. Eisma ${ }^{1 *}$, Rita Rosner ${ }^{2}$ and Hannah Comtesse ${ }^{2}$ \\ ${ }_{1}^{1}$ Department of Clinical Psychology and Experimental Psychopathology, University of Groningen, Groningen, Netherlands, \\ 2 Department of Psychology, Catholic University Eichstaett-Ingolstadt, Ingolstadt, Germany
}

Keywords: prolonged grief disorder, persistent complex bereavement disorder, complicated grief, criteria, algorithm, criticism, critique, multiverse analyses

OPEN ACCESS

Edited by:

Birgit Wagner,

Medical School Berlin, Germany

Reviewed by:

Sebastian Trautmann,

Medical School Hamburg, Germany

Nadine Stammel,

Freie Universität Berlin, Germany

Carina Heeke,

Freie Universität Berlin, Germany

*Correspondence:

Maarten C. Eisma

m.c.eisma@rug.nl

Specialty section:

This article was submitted to

Mood and Anxiety Disorders,

a section of the journal

Frontiers in Psychiatry

Received: 24 February 2020

Accepted: 16 July 2020

Published: 07 August 2020

Citation:

Eisma MC, Rosner $R$ and Comtesse H (2020) ICD-11 Prolonged Grief

Disorder Criteria: Turning Challenges

Into Opportunities With

Multiverse Analyses.

Front. Psychiatry 11:752.

doi: 10.3389/fpsyt.2020.00752

\section{INTRODUCTION}

Recently, prolonged grief disorder (PGD), a diagnosis characterized by severe, persistent and disabling grief, was formally included in the 11th revision of the International Classification of Diseases [ICD-11; (1): Table 1]. To meet PGD $\mathrm{ICD}_{11}$ criteria one needs to experience persistent and pervasive longing for the deceased and/or persistent and pervasive cognitive preoccupation with the deceased, combined with any of 10 additional grief reactions assumed indicative of intense emotional pain for at least six months after bereavement. Contrary to the 5th revision of the Diagnostical and Statistical Manual of Mental Disorders [DSM-5; (11)] and the 10th revision of the International Classification of Diseases [ICD-10; (12)], the ICD-11 only uses a typological approach, implying that diagnosis descriptions are simple and there is no strict requirement for the number of symptoms one needs to experience to meet the diagnostic threshold.

Some researchers have argued that PGD $_{\text {ICD-11 }}$ 's typological approach is helpful, as it will lead to greater sensitivity in case identification in clinical practice and increased cross-cultural applicability (13). Others have highlighted that the typological approach allows for flexible diagnostic algorithms in research, so that $\mathrm{PGD}_{\mathrm{ICD}-11}$ criteria can be adapted to resemble the characteristics of both stricter and more lenient precursor criteria (14). In the current contribution, we take a different, complementary position. We highlight a series of challenges in using the PGD criteria for research purposes and discuss the application of a method that employs flexibility of PGD ICD-11 $_{1}$ diagnostic approach to address these challenges, which may help in working toward the unbiased, structured, and transparent identification of optimal criteria for disturbed grief.

\section{A CRITIQUE OF PGD ICD-11 FOR RESEARCH PURPOSES}

A first challenge to researchers applying $\mathrm{PGD}_{\mathrm{ICD}-11}$ criteria is that they were completely new when first introduced and differed substantially from previously proposed diagnostic criteria sets (15). For example, $\mathrm{PGD}_{\mathrm{ICD}-11}$ contains multiple symptoms not found in any prior proposed criteria set, such as guilt, blame and the inability to experience positive mood [for a full criteria set comparison: (16)]. Furthermore, oft-used measures to assess disturbed grief responses, such as versions of the 
TABLE 1 | Overview of ICD-11 diagnostic criteria for PGD and results of multiverse analyses of PGD ICD-11.

\begin{tabular}{|c|c|c|c|c|}
\hline ICD-11 criteria for PGD & Study & Sample & Measure $^{1}$ & Results \\
\hline $\begin{array}{l}\text { A. At least one of } \\
\text { the following: } \\
\text { a persistent and pervasive } \\
\text { longing for the deceased } \\
\text { or a persistent and } \\
\text { pervasive preoccupation } \\
\text { with the deceased. }\end{array}$ & $\begin{array}{l}\text { Boelen and } \\
\text { Lenferink (2) }\end{array}$ & $\begin{array}{l}855 \\
\text { bereaved } \\
\text { community } \\
\text { members }\end{array}$ & $\begin{array}{l}\text { ICG-R, SCL- } \\
90 \\
\text { depression } \\
\text { scale }\end{array}$ & 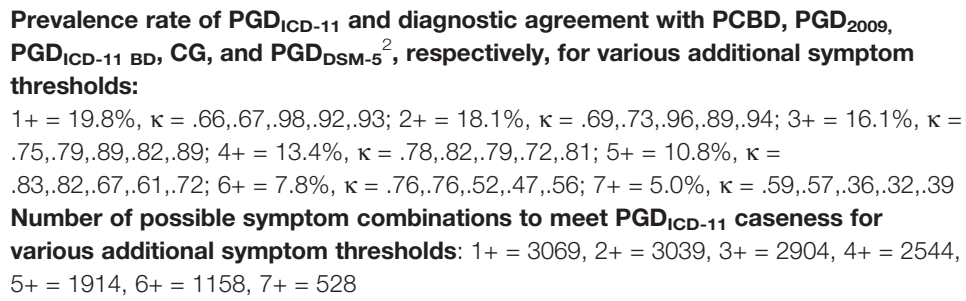 \\
\hline $\begin{array}{l}\text { B. Examples of intense } \\
\text { emotional pain } \\
\text { Accompanied by intense } \\
\text { emotional pain e.g. } \\
\text { sadness, guilt, anger, } \\
\text { denial, blame; difficulty } \\
\text { accepting the death; } \\
\text { feeling one has lost a part }\end{array}$ & $\begin{array}{l}\text { Boelen et al. } \\
(3) \\
\text { Bonanno and } \\
\text { Malgaroli (4) }\end{array}$ & $\begin{array}{l}551 \\
\text { bereaved } \\
\text { community } \\
\text { members } \\
282 \\
\text { bereaved } \\
\text { community } \\
\text { members }\end{array}$ & $\begin{array}{l}\text { ICG-R, SCL- } \\
90 \\
\text { depression } \\
\text { scale } \\
\text { "Structured } \\
\text { clinical } \\
\text { interview" }\end{array}$ & $\begin{array}{l}\text { Prevalence rate of } \mathbf{P G D}_{\mathbf{I C D}-11} \text { and diagnostic agreement with PCBD for various } \\
\text { additional symptom thresholds: } \\
\begin{array}{l}1+=19.2 \%, \kappa=.51,2+=17.6 \%, \kappa=.56 ; 3+=15.4 \%, \kappa=.62 \\
4+=11.1 \%, \kappa=.75 ; 5+=8.3 \%, \kappa=.84,6+=5.3 \%, \kappa=.71\end{array} \\
\text { Prevalence rate of } \mathbf{P G D}_{\mathbf{I C D}-11} \text { and diagnostic agreement with PCBD for various } \\
\text { symptom thresholds: } 1+=11.7 \%, \text { percentage match }=36.4 \%, 3+=8.8 \%, \text { percentage } \\
\text { match }=48.0 \% ; 5+=4.6 \%, \text { percentage match }=76.9 \%\end{array}$ \\
\hline
\end{tabular}
experience positive mood; emotional numbness; difficulty in engaging with social or other activities.

C. Time and impairment Persisted for an abnormally long period of time (more than 6 months at a minimum): following the loss, clearly exceeding expected social, cultural or religious norms for the individual's culture and context.

The disturbance causes significant impairment in personal, family, social, educational, occupational or other important areas of functioning.

$\begin{array}{lll}\text { Comtesse } & 113 & \\ \text { et al. (5) } & \begin{array}{l}\text { bereaved } \\ \text { treatment- } \\ \text { seekers }\end{array} & \\ & \\ & \\ \text { Cozza et al. (6) } & 1732 \\ & \text { bereaved } \\ \text { military family } & \\ \text { members } & \\ \text { Mauro et al. (7) } & \begin{array}{l}\text { 261 } \\ \text { bereaved } \\ \text { treatment- } \\ \text { seekers }\end{array}\end{array}$

\author{
Sensitivity and specificity values from ROC analysis of $P G D_{I C D-11}$ for various additional \\ symptoms thresholds (interview-diagnosed PGD $_{2009}$ as criterion standard): \\ $1+=100 \%$ and $6 \%, 2+=100 \%$ and $9 \%, 3+=100 \%$ and $30 \%$, \\ $4+=98 \%$ and $56 \%, 5+=88 \%$ and $75 \%, 6+=82 \%$ and $92 \%, 7+=65 \%$ and $96 \%, 8+=$ \\ $37 \%$ and $100 \%, 9+=15 \%$ and $100 \%, 10+=7 \%$ and $100 \%$ \\ Prevalence rate of $\mathrm{PGD} \mathrm{ICD}_{\mathrm{I1}}$ and diagnostic agreement with $\mathrm{PCBD}$ for various \\ additional symptom thresholds: $1+=69.1 \%, \kappa=.51 ; 6+=46.9 \%, \kappa=.77$
}

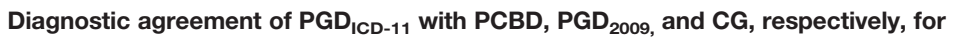 thresholds of one or two additional symptoms for all criteria-sets: $\kappa$ for $P G D_{I C D}-11$ and $\mathrm{PCBD}=.90$ or.87, $\kappa$ for $P \mathrm{PD}_{\mathrm{ICD}-11}$ and $\mathrm{PGD}_{2009}=.89$ or.86, $\kappa$ for $P \mathrm{PD}_{\mathrm{ICD}-11}$ and $\mathrm{CG}$ $=.93$ or.89
Diagnostic agreement of PGD ICD-11 $_{11}$ with ICG cut-off $\geq \mathbf{3 0}$ and clinician-rated CG and functional impairment for various additional symptom thresholds: $0+=95.8 \%$, $1+=95.8 \%$,
$2+=95.0 \%, 3+=91.6 \%, 4+=86.2 \%, 5+=72.8 \%, 6+=45.2 \%(5+$ and $6+$ estimates fall in between $\mathrm{PGD}_{2009}$ rates of agreement: $\left.59.0 \%\right)$

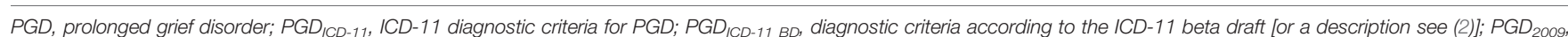
diagnostic criteria for PGD according to Prigerson et al. (8); PCBD, DSM-5 diagnostic criteria for persistent complex bereavement disorder; CG, diagnostic criteria for complicated grief (9); CGQ, Complicated Grief Questionnaire; ICG, Inventory of Complicated Grief; ICG-R, Inventory of Complicated Grief Revised; PG-13, Interview for Prolonged Grief-13; SCI-CG, Structured Clinical Interview for Complicated Grief; SCL-90, Symptom-Checklist-90. ${ }^{1}$ In all cited studies, items of the respective measure(s) were used to assess (approximations of) PGD, symptoms. Symptoms were regarded as present if judged as 'present' by an interviewer (4) or if an item was scored higher than a specific value (e.g., $\geq 4$ ) on the respective scale(s) (e.g., five-point Likert scale). Each symptom score was dichotomously coded as "absent" (0) or "present" (1). PGDICD-11 caseness was then determined following the ICD-11 diagnostic rule.

${ }^{2} P G D_{D S M-5}$ was interpreted here according to proposed guidelines [(10), i.e., three additional symptoms].

Inventory of Complicated Grief [e.g., ICG; (17)] do not fully assess $\mathrm{PGD} \mathrm{ICD}-11_{11}$ criteria [6; for a recent review illustrating this point: (18)]. Therefore, the development of new, reliable and valid instruments is critical to assess the characteristics and validity of $\mathrm{PGD}_{\mathrm{ICD}-11}$ and determine for common research purposes [e.g., establishing prevalence, risk factors, treatment efficacy] who meets $\mathrm{PGD}_{\mathrm{ICD}-11}$ criteria. However, the typological approach in $\mathrm{PGD}_{\mathrm{ICD}-11}$ poses a substantial challenge to the development of such instruments.

First, the plain language used to formulate additional criteria makes it unclear what precisely each criterion implies. Single- word criteria "guilt," "anger," "denial," and "blame" are particularly problematic. For example, "blame" could refer to self-blame or other-blame, blame for the death, or blame for something else. Since self-blame is much more prevalent in bereaved persons than blaming others (19) the interpretation of this criterion influences its prevalence. Moreover, blaming oneself for the death perpetuates disturbed grief, whereas blaming others for the death does not (20), so the characteristics and clinical correlates of this criterion may be very different depending on how it is interpreted [for a related discussion: (21)]. 
Second, even core criteria of "longing" and "preoccupation," shared with most prior proposed grief disorders, are potentially problematic in their implementation. For example, it is unclear if a higher item-score threshold should be used to indicate that someone experiences persistent and pervasive longing, as it is the most frequently reported experience in bereavement (22), and, as a consequence, one of the least sensitive criteria in distinguishing those with and without disturbed grief (14). Preoccupation with the deceased is also already being interpreted differently by influential researchers in the grief field, with some viewing it as intrusive images about the death (23), and others as a process similar to grief rumination (24). While imagery and rumination are related processes, they are dissimilar in phenomenal characteristics, such as their duration, sensory experiences, and emotional correlates (25). The interpretation of this criterion will therefore have repercussions for what we regard as PGD.

Third, many key characteristics of $\mathrm{PGD}_{\mathrm{ICD}-11}$ (e.g., prevalence, classification, symptom heterogeneity) depend heavily on the chosen diagnostic algorithm. Pioneering research on characteristics of this disorder (with measures approximating actual criteria) used the minimal criteria as specified in the ICD-11 (i.e., at least one core criterion, and at least one additional criterion) in addition to the time and disability criteria $(7,26)$. It soon became apparent that applying these minimal criteria led to much higher prevalence rates for PGD ${ }_{\text {ICD-11 }}$ than for prior proposed criteria of PGD [PGD 2009 ; (8)] and persistent complex bereavement disorder [PCBD; DSM-5, (10)]. This algorithm is thus relatively lenient, and applying it may lead to overdiagnosis and limited generalizability of findings on two of the most-studied grief disorder proposals (i.e., $\mathrm{PGD}_{2009}$; PCBD) to

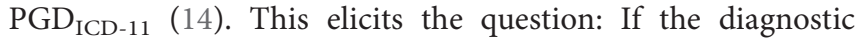
algorithm directly derived from the ICD-11 text is too liberal, which diagnostic rules are then optimal for research?

\section{MULTIVERSE ANALYSES IN RESEARCH ON PGD ICD-11}

In summary, a fundamental challenge for grief researchers in using the $\mathrm{PGD}_{\mathrm{ICD}-11}$ diagnosis is that its criteria are open for multiple interpretations and that the only diagnostic algorithm mapping one-on-one on the diagnosis description is too lenient. While the current criteria cannot easily be amended, their systematic investigation can make them more useful to researchers, for instance by providing a basis for achieving consensus on symptom interpretation, algorithms, and future PGD criteria. We propose that multiverse analyses can be particularly helpful in achieving such goals. Multiverse analyses typically consist of a procedure wherein one performs similar analyses across multiple datasets generated by making reasonable but variable choices on excluding, transforming and coding data (27). For example, when using a reaction time task with skewed data, one may perform analyses based on the median or the mean or analyze the data using parametric or non-parametric statistical tests. By comparing outcomes of multiple analyses, one can establish the degree of uncertainty about the conclusions one arrives at and the robustness of findings to arbitrary decisions made in data preparation and analysis. For example, one may discover that the direction and significance of effects is similar regardless of these decisions or that some decisions lead to significant effects, whereas others do not. The first scenario would allow for strong conclusions and the second scenario would signal caution is warranted in the interpretation of findings.

We advocate a similar but conceptually distinct procedure wherein empirical research examining the characteristics of PGD ${ }_{\text {ICD-11 }}$ systematically vary certain aspects of these criteria (e.g., using a more stringent cut-off for longing) or the diagnostic algorithm (e.g., varying the number of additional symptoms). A comparison of results obtained with multiple interpretations of criteria can help illuminate how robust specific results are dependent on multiple interpretations of the PGD $\mathrm{ICD}_{11}$ criteria. For example, one may be able to investigate the robustness of group differences between people with and without PGD on risk-factors and protective factors or treatment effectiveness (e.g., percentage with and without diagnosis after treatment) dependent on different interpretations of $\mathrm{PGD}_{\mathrm{ICD}-11}$. Additionally, critical information can be gathered on the influence of variations in symptom interpretations and algorithms on $\mathrm{PGD}_{\mathrm{ICD}-11}$ characteristics and how these characteristics compare to other proposed criteria sets [e.g., the newly developed PGD criteria for the upcoming text revision of DSM-5, (10)]. That is, multiverse analyses can be applied to shed light on a variety of clinically relevant characteristics of $\mathrm{PGD}_{\mathrm{ICD}-11}$ (e.g., retest and interrater reliability, specificity and sensitivity of classification, distinctiveness from other disorders, associations with functional impairment) when systematically modifying interpretations of its criteria.

Only a handful of studies have thus far applied such analyses, which have predominantly narrowly focused on examining characteristics of $\mathrm{PGD}_{\mathrm{ICD}-11}$ and their comparability against external standards when varying the number of additional criteria (see Table 1 for a summary). It has been observed that minimal PGD $\mathrm{ICD}-11_{11}$ criteria yield a similar prevalence as the relatively lenient Shear et al. criteria (9) for complicated grief (7), but almost two times higher prevalence than relatively strict PCBD criteria $(2,28,29)$. Multiverse analyses in community samples demonstrated that similar prevalence estimates and good diagnostic agreement with $\mathrm{PCBD}$ and $\mathrm{PGD}_{2009}$ appears to be achieved with five additional criteria for PGD $\mathrm{ICD}-11_{1}[(2-4)$, cf. (7)]. Similarly, in treatment-seekers, minimal PGD ICD-11 $_{1}$ criteria correctly classified people against a relatively lenient standard of 30 or higher on the ICG $(6,7)$, yet as many as six additional symptoms were necessary to yield comparable prevalence and good diagnostic agreement with $\mathrm{PGD}_{2009}$ and PCBD (5). Moreover, one study demonstrated the influence of the number of additional symptoms on symptom heterogeneity, theoretically demonstrating that the number of ways to meet PGD ICD-11 criteria ranges from 3.069 for one to 528 for seven additional symptoms (2). A less heterogeneous diagnosis is clearly preferable, as it would lead to less variability within groups of people meeting grief disorder criteria, making the distinction between these people more useful for research and practice (30). Taken together, these examples illustrate that the 
properties of $\mathrm{PGD}_{\mathrm{ICD}-11}$ depend on both the chosen diagnostic rule and the stringency of the comparison standard, and that the number of additional symptoms is critical in determining prevalence, clinical classification, and symptom profiles.

For future research, we recommend multiverse analyses varying not only the algorithm, but additionally symptom interpretations of single-item criteria and cognitive preoccupation and cut-offs for the presence of the longing criterion. We also advise to substantially expand the current focus of multiverse analyses of PGD ICD-11 to establish the robustness of clinically-relevant findings (e.g., on treatment efficacy) and the variety of other aspects relevant to the validity of a diagnosis [for reviews: $(13,24)$ ]. The latter includes - but is not limited to: reliability of classification [e.g., (31)], the structure of symptoms [e.g., (32)], distinctiveness from related disorders [e.g., (33)], and relationships with functional impairment [e.g., (34)]. We further advocate transparency in applying multiverse analyses and recommend: open access publication, data accessibility (e.g., through availability in repositories), fully specifying the origins and formulations of items used to assess $\mathrm{PGD}_{\mathrm{ICD}-11}$ and, if applicable, other disturbed grief criteria, and complete reporting of the variations of $\mathrm{PGD}_{\mathrm{ICD}-11}$ and outcomes under investigation.

\section{DISCUSSION}

In the absence of clearly defined criteria and diagnostic rules for PGD $\mathrm{ICD}_{\mathrm{I} 11}$, researchers should broadly apply structured methods to examine the characteristics of this disorder and compare it against past and future proposed grief disorders. Multiverse analyses can be a powerful tool to determine the validity and clinical usefulness of

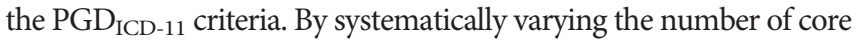
and additional symptoms, the interpretation of symptoms, and the

\section{REFERENCES}

1. World Health Organization. ICD-11 beta draft website [Information]. (2018). Available at: https://icd.who.int/browse11/1-m/en (Accessed on 24th June 2020).

2. Boelen PA, Lenferink LIM. Comparison of six proposed diagnostic criteria sets for disturbed grief. Psychiatry Res (2020) 285:112786. doi: 10.1016/ j.psychres.2020.112786

3. Boelen PA, Lenferink LIM, Smid GE. Further evaluation of the factor structure, prevalence, and concurrent validity of DSM-5 criteria for persistent complex bereavement disorder and ICD-11 criteria for prolonged grief disorder. Psychiatry Res (2019a) 3:206-10. doi: 10.1016/j.psychres.2019.01.006

4. Bonanno GA, Malgaroli M. Trajectories of grief: Comparing symptoms from the DSM-5 and ICD-11 diagnoses. Depress Anxiety (2020) 37:17-25. doi: 10.1002/da.22902

5. Comtesse H, Vogel A, Kersting A, Rief W, Steil R, Rosner R. When does grief become pathological? Evaluation of the ICD-11 diagnostic proposal for prolonged grief in a treatment-seeking sample. Eur J Psychotraumatol (2020) 11:1694348. doi: 10.1080/20008198.2019.1694348

6. Cozza SJ, Shear MK, Reynolds CF, Fisher JE, Zhou J, Maercker A, et al. Optimizing the clinical utility of four proposed criteria for a persistent and impairing grief disorder by emphasizing core, rather than associated symptoms. Psychol Med (2020) 50:438-45. doi: 10.1017/S003329 1719000254 standards for meeting symptoms, we can evaluate how such decisions influence the characteristics of $\mathrm{PGD}_{\mathrm{ICD}-11}$, also in relation to different external standards. This will create a comprehensive research base enabling us to enhance our understanding of $\mathrm{PGD}_{\mathrm{ICD}-11}$ and of disturbed grief more generally. Creating this research base is no panacea: it cannot undo the inherent weaknesses of the PGD $\mathrm{ICD}_{11}$ criteria. However, the systematic evaluation of this information will help clarify under which circumstances diagnoses behave similarly or differently, providing a stepping stone to harmonize PGD ICD-11 $_{11}$ criteria with other criteria sets and to develop more optimal future disturbed grief criteria.

\section{AUTHOR CONTRIBUTIONS}

All authors developed the ideas for this article. ME and HC wrote the first draft of the manuscript. RR contributed important intellectual content by providing critical revisions to the first draft.

\section{FUNDING}

ME was supported by a Netherlands Organization for Scientific Research (NWO) Veni grant [Grant ID: 016.veni195.113]. The funder did not play a role in the study design, the writing of the report or in the decision to submit the article for publication. RR was supported by a grant of Deutsche Forschungsgemeinschaft [Grant ID: RO2042/7-1]. The funder did not play a role in the study design, the writing of the report or the decision to submit the article for publication.
7. Mauro C, Reynolds CF, Maercker A, Skritskaya N, Simon N, Zisook S, et al. Prolonged grief disorder: Clinical utility of ICD-11 diagnostic guidelines. Psychol Med (2019) 49:861-7. doi: 10.1017/S0033291718001563

8. Prigerson HG, Horowitz MJ, Jacobs SC, Parkes CM, Aslan M, Goodkin K, et al. Prolonged grief disorder: Psychometric validation of criteria proposed for DSM-V and ICD-11. PloS Med (2009) 6:e1000121. doi: 10.1371/ journal.pmed.1000121

9. Shear MK, Simon N, Wall M, Zisook S, Neimeyer R, Duan N, et al. Complicated grief and related bereavement issues for DSM-5. Depress Anxiety (2011) 28:103-17. doi: 10.1002/da.20780

10. American Psychiatric Association. View and comment on recently proposed changes to DSM-5. (2020). Available at: https://www.psychiatry.org/ psychiatrists/practice/dsm/proposed-changes (Accessed on 24th of June 2020).

11. American Psychiatric Association. Diagnostic and statistical manual of mental disorders. 5th ed. American Psychiatric Publishing: Arlington, VA (2013).

12. World Health Organization. ICD-10: international statistical classification of diseases and related health problems: tenth revision. 2nd ed. Geneva, Switzerland: World Health Organization (2004).

13. Killikelly C, Maercker A. Prolonged grief disorder for ICD-11: The primacy of clinical utility and international applicability. Eur J Psychotraumatol (2017) 8:1476441. doi: 10.1080/20008198.2018.1476441

14. Simon NM, Wall MM, Keshaviah A, Dryman MT, LeBlanc NJ, Shear MK. Informing the symptom profile of complicated grief. Depress Anxiety (2011) 28:118-26. doi: 10.1002/da.20775 
15. Eisma MC, Lenferink LIM. Response to: Prolonged grief disorder for ICD-11: The primacy of clinical utility and international applicability. Eur J Psychotraumatol (2017) 8:1512249. doi: 10.1080/20008198.2018. 1512249

16. Lenferink LIM, Boelen PA, Smid G, Paap M. The importance of harmonising diagnostic criteria sets for pathological grief. Brit J Psychiat (in press), 1-4. doi: 10.1192/bjp.2019.240

17. Prigerson HG, Maciejewski PK, Reynolds CF,IIIII, Bierhals AJ, Newsom JT, Fasiczka A, et al. Inventory of complicated grief: A scale to measure maladaptive symptoms of loss. Psychiatry Res (1995) 59:65-79. doi: 10.1016/0165-1781(95)02757-2

18. Treml J, Kaiser J, Plexnies A, Kersting A. Assessing prolonged grief disorder: A systematic review of assessment instruments. J Affect Disord (2020) 274:42034. doi: $10.1016 /$ j.jad.2020.05.049

19. Davis CG, Lehman DR, Wortman CB, Silver RC, Thompson SC. The undoing of traumatic life events. Pers Soc Psychol Bull (1995) 21:109-24. doi: 10.1177/ 0146167295212002

20. Eisma MC, Epstude K, Schut HAW, Stroebe MS, Simion A, Boelen PA. Upward and downward counterfactuals after loss: A multi-wave controlled longitudinal study. Behav Ther (in press). doi: 10.1016/ j.beth.2020.07.007

21. Greene T. Blame, PTSD and DSM-5: An urgent need for clarification. Eur J Psychotraumatol (2018) 9:1468709. doi: 10.1080/20008198.2018.1468709

22. Maciejewski PK, Zhang B, Block SD, Prigerson HG. An empirical examination of the stage theory of grief. JAMA (2007) 297:716-23. doi: 10.1001/ jama.297.7.716

23. Boelen PA, Smid GE. The Traumatic Grief Inventory Self-Report Version (TGI-SR): Introduction and preliminary psychometric evaluation. $J$ Loss Trauma (2017) 22:196-212. doi: 10.1080/15325024.2017.1284488

24. Simon NM, Shear MK, Reynolds CF, Cozza SJ, Mauro C, Zisook S, et al. Commentary on evidence in support of a grief-related condition as a DSM diagnosis. Depress Anxiety (2020) 37:9-16. doi: 10.1002/da.22985

25. Speckens AE, Ehlers A, Hackmann A, Ruths FA, Clark DM. Intrusive memories and rumination in patients with post-traumatic stress disorder: A phenomenological comparison. Mem (2007) 15:249-57. doi: 10.1080/ 09658210701256449

26. Boelen PA, Lenferink LIM, Nickerson A, Smid GE. Evaluation of the factor structure, prevalence, and validity of disturbed grief in DSM- 5 and ICD- 11. J Affect Disord (2018) 240:79-87. doi: 10.1016/j.jad.2018.07.041
27. Steegen S, Tuerlinckx F, Gelman A, Vanpaemel W. Increasing transparency through a multiverse analysis. Perspect Psychol Sci (2016) 11:702-12. doi: $10.1177 / 1745691616658637$

28. Boelen PA, Spuij M, Lenferink LII. Comparison of DSM-5 criteria for persistent complex bereavement disorder and ICD-11 criteria for prolonged grief disorder in help-seeking bereaved children. J Affect Disord (2019b) 250:71-8. doi: 10.1016/j.jad.2019.02.046

29. O'Connor M, Lasgaard M, Larsen L, Johannsen M, Lundorff M, FarverVestergaard I, et al. Comparison of proposed diagnostic criteria for pathological grief using a sample of elderly bereaved spouses in Denmark: Perspectives on future bereavement research. J Affect Disord (2019) 251:52-9. doi: 10.1016/j.jad.2019.01.056

30. Lenferink LIM, Eisma MC. 37.650 ways to have "persistent complex bereavement disorder" yet only 48 ways to have "prolonged grief disorder". Psychiatry Res (2018) 261:88-9. doi: 10.1016/j.psychres.2017.12.050

31. Lichtenthal WG, Maciejewski PK, Demirjian CC, Roberts KE, First MB, Kissane DW, et al. Evidence of the clinical utility of a prolonged grief disorder diagnosis. World Psychiatry (2018) 17:364-5. doi: 10.1002/wps.20544

32. Golden A-M, Dalgleish T. Is prolonged grief distinct from bereavementrelated posttraumatic stress? Psychiatry Res (2010) 178:336-41. doi: 10.1016/ j.psychres.2009.08.021

33. Malgaroli M, Maccallum F, Bonanno G. Symptoms of persistent complex bereavement disorder, depression, and PTSD in a conjugally bereaved sample: A network analysis. Psychol Med (2018) 48:1-10. doi: 10.1017/S003329 1718001769

34. Maccallum F, Bryant RA. A network approach to understanding quality of life impairments in prolonged grief disorder. J Trauma Stress (2020) 31:106-15. doi: $10.1002 /$ jts.22383

Conflict of Interest: The authors declare that the research was conducted in the absence of any commercial or financial relationships that could be construed as a potential conflict of interest.

Copyright (๑) 2020 Eisma, Rosner and Comtesse. This is an open-access article distributed under the terms of the Creative Commons Attribution License (CC BY). The use, distribution or reproduction in other forums is permitted, provided the original author(s) and the copyright owner(s) are credited and that the original publication in this journal is cited, in accordance with accepted academic practice. No use, distribution or reproduction is permitted which does not comply with these terms. 